INPLASY

PROTOCOL

To cite: Li et al. Evaluation of the effectiveness and safety of acupuncture in the treatment of premature ventricular contractions: A protocol for systematic review and metaanalysis. Inplasy protocol 2021100040. doi: 10.37766/inplasy2021.10.0040

Received: 13 October 2021

Published: 13 October 2021

Corresponding author: Yinghui Liu

1029906143@qq.com

Author Affiliation: Changchun University of Chinese Medicine.

Support: No.

Review Stage at time of this submission: The review has not yet started.

Conflicts of interest: None declared.

\section{Evaluation of the effectiveness and safety of acupuncture in the treatment of premature ventricular contractions: A protocol for systematic review and meta-analysis}

Li, HY1; Liu, AD2; Liu, ZH³; Cheng, GY4; Cui, JF5; Li, SD6; Li, PF7; Xin, YN8; Liu, YH

Review question / Objective: The purpose of this study is to explore the efficacy of acupuncture in the treatment of patients with premature ventricular contractions and to provide an effective reference for clinicians and patients on its use.

Information sources: This study was prepared in accordance with the guidelines of the Preferred Reporting Items for Systematic Reviews and Meta-analyses Protocols (PRISMA$P)$. This protocol is meant for a systematic review and metaanalysis of previously published studies, so it does not require patient and public participation, nor does it require ethical approval. We will search foreign language literature databases such as PubMed, Embase, Web of Science, and the Cochrane Library as well as Chinese literature databases such as the China National Knowledge Infrastructure, Wanfang Database, China Science Journal Database, and China Biomedical Literature Database. using the keywords "acupuncture" and "premature ventricular contractions." Our aim is to find randomized clinical trial (RCT) articles on the use of acupuncture in the treatment of PVCs. The search time frame was from the establishment of each of the databases through October 10, 2021.

INPLASY registration number: This protocol was registered with the International Platform of Registered Systematic Review and Meta-Analysis Protocols (INPLASY) on 13 October 2021 and was last updated on 13 October 2021 (registration number INPLASY2021100040).

\section{INTRODUCTION}

Review question / Objective: The purpose of this study is to explore the efficacy of acupuncture in the treatment of patients with premature ventricular contractions and to provide an effective reference for clinicians and patients on its use. 
Condition being studied: Premature ventricular contractions (PVCs) are heartbeats that occur below the bifurcation of the His bundle, prematurely depolarizing the myocardium. [1] They are the most common arrhythmia. Among the general population, their prevalence on conventional electrocardiograms is estimated at 1-4\%. [2] PVCs are common in various organic heart diseases, such as coronary heart disease, rheumatic heart disease, cardiomyopathy, etc. but also in nonorganic heart disease, such as autonomic dysfunction, emotional stress, electrolyte imbalance, etc. [3] The clinical symptoms of PVCs have great variability, ranging from asymptomatic and mild heart palpitations to premature beats triggering malignant ventricular arrhythmia and causing syncope, or blackouts, which seriously affect people's lives and work. [4] At present, the commonly used drugs for the treatment of PVCs in Western medicine include betablockers, mexiletine, and amiodarone, etc. These drugs can correct the original arrhythmia, but they can also induce new arrhythmias, thus affecting the treatment effect of patients, and should not be taken for long periods of time. [5] In contrast, traditional Chinese medicine offers obvious advantages. Existing studies have shown that acupuncture, Chinese patent medicines, and Chinese herbal medicines have significant positive effects in treating PVCs, not only regulating the overall function of the patient's body but also reducing the side effects experienced with Western medicine. [6-10] A systematic review of the treatment of PVCs with traditional Chinese medicine has been published, but there is no systematic review of the effectiveness of acupuncture in the treatment of PVCs. Therefore, we conducted this research to provide a scientific reference for the alternative treatment of PVCs using acupuncture.

\section{METHODS}

Participant or population: The patients met the internationally recognized diagnostic criteria for PVCs, regardless of race, age and sex.
Intervention: The experimental group received acupuncture treatment alone or acupuncture combined with treatment by conventional Western medicine.

Comparator: The control group was a blank control group, a placebo group, or received treatment only through conventional Western medicine.

Study designs to be included: Randomized clinical trial.

Eligibility criteria: The literature included in our study met the following criteria: the patients met the internationally recognized diagnostic criteria for PVCs, regardless of race, age and sex; the study type was an $\mathrm{RCT}$; the experimental group received acupuncture treatment alone or acupuncture combined with treatment by conventional Western medicine; and the control group was a blank control group, a placebo group, or received treatment only through conventional Western medicine. Exclusion criteria were as follows: those studies with participants who did not meet the diagnostic criteria of PVCs or with participants with severe complications; non-RCTs research; studies with duplicate data or incomplete data; case reports, animal experiments, research protocols, reviews, and conference summaries, etc.

Information sources: This study was prepared in accordance with the guidelines of the Preferred Reporting Items for Systematic Reviews and Meta-analyses Protocols (PRISMA-P). This protocol is meant for a systematic review and metaanalysis of previously published studies, so it does not require patient and public participation, nor does it require ethical approval. We will search foreign language literature databases such as PubMed, Embase, Web of Science, and the Cochrane Library as well as Chinese literature databases such as the China National Knowledge Infrastructure, Wanfang Database, China Science Journal Database, and China Biomedical Literature Database. using the keywords "acupuncture" and "premature ventricular contractions." Our aim is to find 
randomized clinical trial (RCT) articles on the use of acupuncture in the treatment of PVCs. The search time frame was from the establishment of each of the databases through October 10, 2021.

Main outcome(s): The primary outcome indicators were clinical symptom curative effects, ECG curative effects, and total number of PVCs in $24 \mathrm{~h}$ dynamic electrocardiograms.

Additional outcome(s): Secondary outcome indicators were Traditional Chinese Medicine syndrome curative effects, Traditional Chinese Medicine syndrome scores, heart rate improvement, and adverse reactions.

Quality assessment / Risk of bias analysis: Two researchers (PL and $Y X$ ) will independently apply the bias risk assessment tool provided by Cochrane System Reviewer Manual 5.1.0 to evaluate the quality of the included research literature. Specific content to be assessed includes random sequence generation, allocation hiding, blinding of patients, researchers and outcome evaluators, completeness of results data, selective reporting and other biases. These studies will be divided into three quality levels: high risk of bias, low risk of bias, and unclear risk of bias. If there is a disagreement, it will be resolved through internal negotiation or through discussion with a third researcher $(\mathrm{YL})$.

Strategy of data synthesis: We will use RevMan5.3 software to analyze the data. The effect value of the count data uses the relative risk (RR), and the effect value of the measurement data uses the standardized mean difference (SMD), which is represented by a $95 \%$ confidence interval (Cl). We will use a $P$ value and 12 to evaluate the heterogeneity in the results of the study: $\mathbf{1 2 0 . 0 5}$ indicates that the level of heterogeneity is low, and the fixed effects model will be used for analysis; $12 \geq 50 \%$ and $P \leq 0.05$ indicates existence significant heterogeneity, and the select random effects model will be used for analysis. Subgroup analysis or sensitivity analysis will be performed according to the possible causes of heterogeneity.

Subgroup analysis: We will conduct a subgroup analysis of factors that may lead to sources of heterogeneity, such as the number of cases included in the literature, age, intervention measures, treatment types, and duration of treatment.

Sensitivity analysis: If possible, we will conduct a sensitivity analysis to assess the robustness of the included results. If the results are unstable, studies with a high risk of bias will be excluded.

Country(ies) involved: China.

Other relevant information: References: [1] Rujirachun P, Wattanachayakul P, Phichitnitikorn $P$, et al. Association of premature ventricular complexes and risk of ischemic stroke: A systematic review and meta-analysis. Clin Cardiol. 2021;44:151-159. [2] Huizar JF, Tan AY, Kaszala K, et al. Clinical and translational insights on premature ventricular contractions and PVC-induced cardiomyopathy. Prog Cardiovasc Dis. 2021;66: 17-27. [3] Marcus GM. Evaluation and Management of Premature Ventricular Complexes. C irculation. 2020;141:1404-1418. [4] Kim YG, Han KD, Choi JI, et al. Premature ventricular contraction is associated with increased risk of atrial fibrillation: a nationwide population-based study. Sci Rep. 2021;11:1601. [5] Zhang F,Zou J,Yu H et al. Acute Efficacy of a Traditional Chinese Medicine for Treatment of Frequent Premature Ventricular Contractions in Patients with Concomitant Sinus Bradycardia: Results from a Double-Blind, Placebo-Controlled, Multicentre, Randomized Clinical Trial. Evid Based Complement Alternat Med. 2019; 2019: 3917282. [6] Liu J, Li SN, Liu L, et al. Conventional Acupuncture for Cardiac Arrhythmia: A Systematic Review of Randomized Controlled Trials. Chin J Integr Med. 2018;24:218-226. [7] Li Y,BarajasMartinez H,Li B et al. Comparative Effectiveness of Acupuncture and Antiarrhythmic Drugs for the Prevention of 
Cardiac Arrhythmias: A Systematic Review and Meta-analysis of Randomized Controlled Trials.Front Physiol. 2017;8:358. [8] Han B, Wang F. Acupuncture treatment for 98 cases of ventricular premature beat. J Tradit Chin Med. 2008;28(2):86-9. [9] Zou JG, Zhang J, Jia ZH, et al. Evaluation of the traditional Chinese Medicine Shensongyangxin capsule on treating premature ventricular contractions: a randomized, double-blind, controlled multicenter trial. Chin Med J. 2011;124:76-83. [10] Hua W, Gao RL, Zhao BC, et al. The Efficacy and Safety of Wenxin Keli in Patients with Frequent Premature Ventricular Contractions: A Randomized, Double-blind, Placebo-controlled, Parallelgroup, Multicenter Trial. Chin Med J. 2015;128:2557-64.

Keywords: acupuncture, premature ventricular beats, protocol, systematic review, meta-analysis.

Contributions of each author:

Author 1 - Hongyu Li.

Author 2 - Aidong Liu.

Author 3 - Zhenhua Liu.

Author 4 - Guangyu Cheng.

Author 5 - Junfeng Cui.

Author 6 - Shuangdi Li.

Author 7 - Pengfei Li.

Author 8 - Yuning Xin.

Author 9 - Yinghui Liu. 